\title{
Post-Finasteride Adverse Effects in Male Androgenic Alopecia: A Case Report of Vitiligo
}

\author{
Ion G. Motofei ${ }^{a}$ David L. Rowland ${ }^{e}$ Simona R. Georgescu ${ }^{b}$ Mircea Tampa ${ }^{b}$ \\ Stana Paunica $^{b}$ Vlad D. Constantin ${ }^{b}$ Cristian Balalau $^{b}$ Mirela Manea $^{c}$ \\ Bogdan C. Baleanu ${ }^{f}$ loanel Sinescu ${ }^{d}$ \\ Departments of a Surgery/Dermatology, ${ }^{b}$ Dermatology, ${ }^{c}$ Psychiatry and d Urology, Carol Davila University,

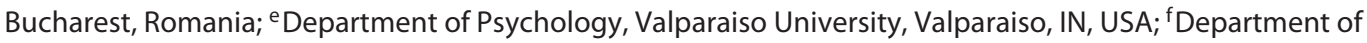 \\ Urology, Hermann-Josef-Krankenhaus, Erkelenz, Germany
}

\section{Keywords}

Male androgenic alopecia · Post-finasteride syndrome .

Vitiligo · Depression · Sexual dysfunctions

\begin{abstract}
Finasteride has proved to be relatively safe and effective in the therapeutic management of male androgenic alopecia. However, literature data report several endocrine imbalances inducing various adverse effects, which often persist after treatment cessation in the form of post-finasteride syndrome. Here we present the case of a 52-year-old man receiving finasteride (1 $\mathrm{mg} /$ day) who developed an uncommon adverse effect represented by generalized vitiligo 2 months after finasteride discontinuation. Associated adverse effects encountered were represented by mild sexual dysfunction (as determined by the International Index of Erectile Function, IIEF) and moderate depressive symptoms (according to DSM-V criteria), all of these manifestations aggregating within/as a possible post-finasteride syndrome. Further studies should develop and compare several therapeutic approaches, taking into account not only compounds that decrease the circulating dihydrotestosterone
\end{abstract}

\section{KARGER}

(C) 2017 S. Karger AG, Basel

E-Mail karger@karger.com

www.karger.com/spp level but also those that could block the dihydrotestosterone receptors (if possible, compounds with selective tropism towards the skin). In addition, the possibility of predicting adverse effects of finasteride (according to hand preference and sexual orientation) should be taken into account.

(c) 2017 S. Karger AG, Basel

\section{Introduction}

Male androgenic alopecia is a moderately stressful condition, which often leads to medical treatment due to an alteration of body image satisfaction. Early stages of hair loss can be stopped or even reversed through 5 - $\alpha$-reductase inhibitors like finasteride, with proven efficacy and relative safety based on the research literature. The same studies, however, describe a number of adverse effects based on finasteride use, most often manifested in psychiatric (depression), genitourinary (sexual arousal and libido disorders), endocrine (breast enlargement), dermatological (rash, pruritus, melasma, etc.), and oral (gingival hypertrophy, etc.) problems [1-3]. 


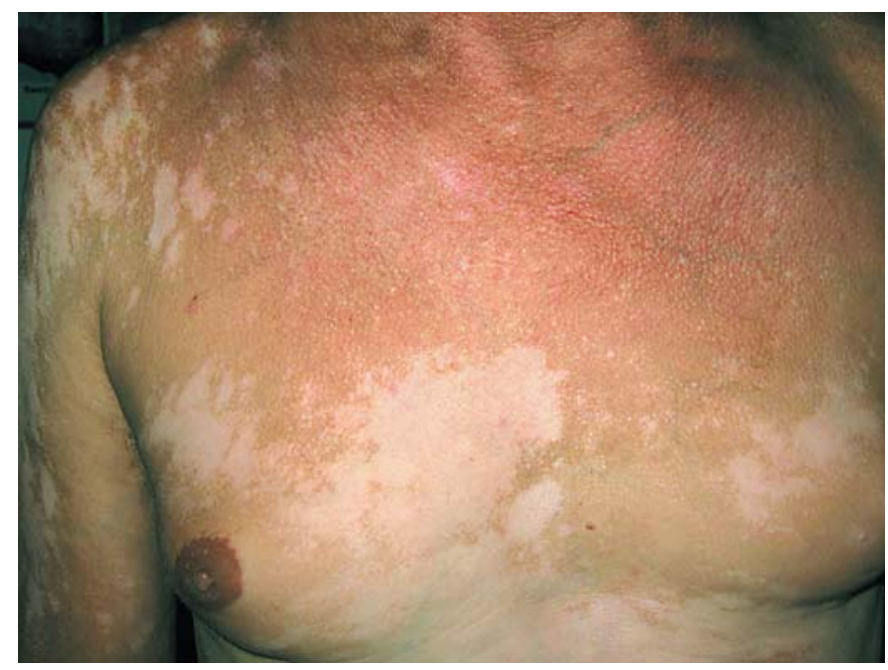

Fig. 1. Gynecomastia and vitiligo.

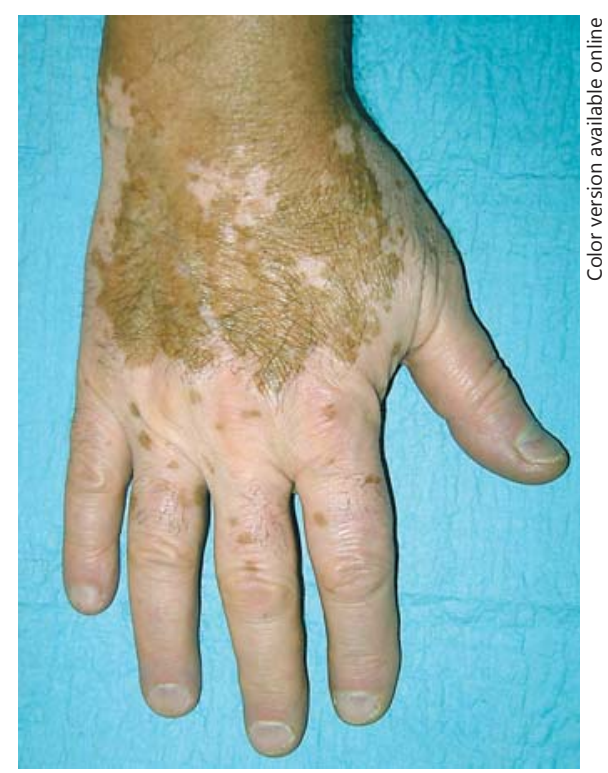

Fig. 2. Vitiligo on the dorsal hand.

Our previous reports are related to relatively common adverse effects encountered on a sample of men treated with finasteride, and are mainly related to dermatological, sexual, and psychiatric impairments [3, 4]. In this paper we present the case of a subject treated with finasteride for male androgenic alopecia who, 2 months after finasteride cessation, developed an uncommon adverse effect represented by generalized vitiligo (white blotchy patches of skin), which was included by us as a post-finasteride syndrome.

Vitiligo after Finasteride Administration

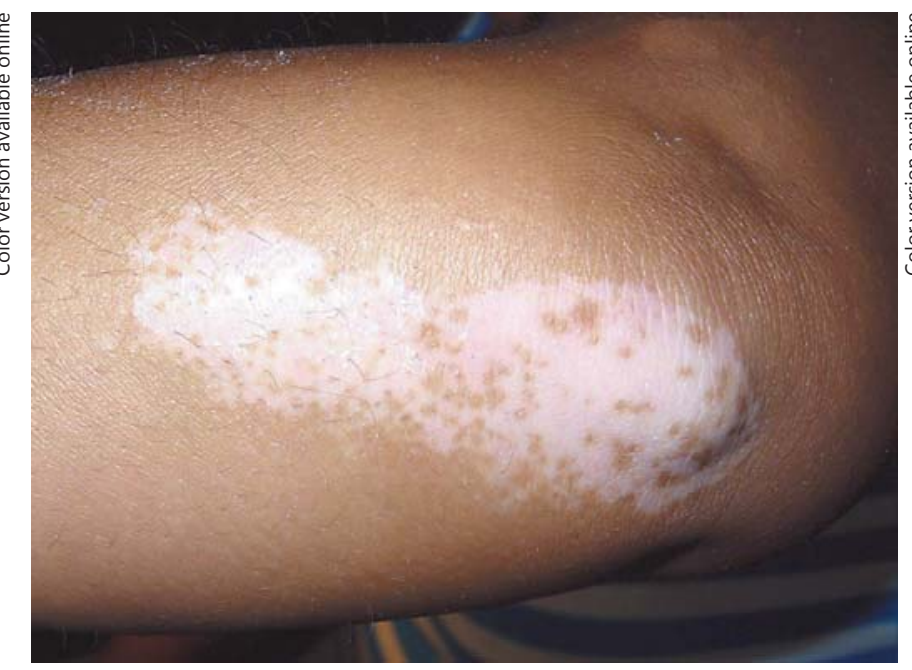

Fig. 3. Vitiligo on the forearm.

\section{Case Report}

A 52-year-old man enrolled in our sample with male androgenic alopecia received finasteride $1 \mathrm{mg}$ /day for a period of 4 months. Inclusion criteria were represented by the existence of a reasonably high level of sexual functioning (as determined by the International Index of Erectile Function, IIEF), the absence of psychiatric impairment (according to DSM-V criteria), and the absence of other general or local affections. Hormonal dosages performed before the study (normal plasmatic values for testosterone, dihydrotestosterone, and estradiol derivates) were also included in the initial selection criteria.

After treatment cessation this patient was assessed again, completing the IIEF questionnaire (which revealed mild erectile dysfunction), according to DSM-V criteria for depression (which indicated moderate depressive symptoms). Hormonal plasmatic levels also showed changes, with a significant decrease in dihydrotestosterone - the most active androgenic compound (about $70 \%)$ - and a slight increase in circulating estradiol. Clinical examination revealed bilateral gynecomastia (Fig. 1), most probably due to finasteride-induced androgen-estrogen imbalances.

Two months after finasteride cessation the patient presented with generalized vitiligo (Fig. 2, 3) and persistent sexual and depressive side effects. These symptoms remained unchanged following subsequent observation for the next 3 months. In response to finasteride, the subject registered a slight improvement (hair density/thickness) after the 4 therapeutic months, and this effect was partly lost 2 months later when the subject complained of vitiligo.

\section{Discussion}

Vitiligo is a chronic disease represented by depigmentation of the skin in the form of asymptomatic whitish patches or macule. Its etiology remains unknown, al- 
though autoimmune factors related to specific genetic mutations are suspected. Consequently, autoimmune diseases like halo nevi, autoimmune thyroid diseases, etc. have been found to occur with greater frequency in subjects with vitiligo $[5,6]$.

Taking into account the potential autoimmune substrate for vitiligo, we took note of 2 distinct neuroendocrine disorders in our patient that might have been contributing to, or at least be related to, the possible neuroendocrine/autoimmune reactions responsible for vitiligo.

The first factor noted was depression. According to the literature, finasteride treatment leads to a decrease in $\alpha$-reduced and an increase in $5 \beta$-reduced and $7 \alpha$-hydroxy metabolites - neuroactive steroids modulating GABA and NMDA receptors in the brain. During finasteride administration, the decrease of circulating steroids with inhibitory GABA activity contributes to several manifestations, like the occurrence of depression in some patients and/or a decrease in alcohol consumption $[1,7]$. In fact, the research literature reports that up to $50 \%$ of patients with vitiligo develop various depressive symptoms following a vitiligo occurrence/diagnosis [8]. It is still unclear to what extent depression appears secondary to vitiligo (due to an alteration of body image) and/or to what degree vitiligo occurs to patients predisposed to depression (with preexisting subclinical depression). In support of this latter viewpoint, recent studies show that depression and immunity could be strongly interrelated within neuroimmune mechanisms [9].

The second factor is represented by endocrine disturbances, manifested clinically in the case of this patient as gynecomastia. Our records, along with the research literature, show that altered levels of neuroactive steroids (associated with depression symptoms) are present in patients with alopecia a long time after the discontinuation of finasteride treatment $[1,10]$. Considering possible interrelations among finasteride, endocrine disturbances, and vitiligo, it is well known that estrogens can enhance immune response, thus playing perhaps important roles in several autoimmune (rheumatic, thyroid, etc.) diseases [11]. On the other hand, melisma - a hypermelanosis representing an effect opposite to vitiligo - is also related to the action of estrogens and progesterone on melanocytes, and has been encountered in some studies as a side effect of finasteride [2].

Finally, we cannot exclude a possible direct action of finasteride on some general or cutaneous neuroendocrine circuits/systems, which could be related to physiopathological mechanisms of vitiligo.

\section{Conclusion}

Subjects with male androgenic alopecia treated with finasteride can develop several adverse effects, which are variable with respect to symptomatology, frequency of occurrence, and persistence. Depression and sexual dysfunction are the most often described persistent adverse effects after finasteride administration. As a consequence, these persistent/posttherapeutic adverse effects have been defined as a new pathological entity, post-finasteride syndrome. Usually, this syndrome is established at 3 or 4 months after finasteride cessation. Our patient developed vitiligo at 2 months following the termination of finasteride therapy - presumed in our opinion as a post-finasteride syndrome. It is yet unclear whether this observed posttherapeutic adverse effect of finasteride is very rare or, alternatively, whether it is in part underestimated due to the discontinuation of treatment and subsequent discontinuation of monitoring and reporting.

Further therapeutic approaches to male androgenic alopecia should perhaps take into account, if possible, compounds that block the dihydrotestosterone receptors (preponderantly to the skin level) rather than compounds that decrease the circulating dihydrotestosterone level. However, the possibility of predicting adverse effects of finasteride, where applicable, should also be taken into account $[4,12]$.

\section{Acknowledgment}

This case report resulted from a scientific project of the Ministry of National Education, CNCS-UEFISCDI (project No. PN-IIID-PCE-2012-4-0409).

\section{Statement of Ethics}

The study sample (http://www.scholar.ro/finasteride) containing the subject was established after obtaining informed consent from each participant, and the study protocol was approved by our institute's committee on human research.

\section{Disclosure Statement}

All authors confirm that there is no conflict of interest to declare.
44

Skin Pharmacol Physiol 2017;30:42-45 DOI: $10.1159 / 000455972$
Motofei et al. 


\section{References}

1 Ali AK, Heran BS, Etminan M: Persistent sexual dysfunction and suicidal ideation in young men treated with low-dose finasteride: a pharmacovigilance study. Pharmacotherapy $2015 ; 35: 687-695$.

2 Famenini S, Gharavi NM, Beynet DP: Finasteride associated melasma in a Caucasian male. J Drugs Dermatol 2014;13:484-486.

3 Paunica S, Giurgiu M, Vasilache A, Paunica I, Motofei I, Dumitriu HT, Dumitriu AS: Finasteride adverse effects and post-finasteride syndrome; implications for dentists. J Mind Med Sci 2016;3:71-79.

4 Motofei IG, Rowland DL, Georgescu SR, Tampa M, Baconi D, Stefanescu E, Baleanu BC, Balalau C, Constantin V, Paunica S: Finasteride adverse effects in subjects with androgenic alopecia: a possible therapeutic approach according to the lateralization process of the brain. J Dermatolog Treat 2016;4:1-3.
5 Patrizi A, Bentivogli M, Raone B, Dondi A, Tabanelli M, Neri I: Association of halo nevus/i and vitiligo in childhood: a retrospective observational study. J Eur Acad Dermatol Venereol 2013;27:148-152.

6 Gong Q, Li X, Gong Q, Zhu W, Song G, Lu Y: Hashimoto's thyroiditis could be secondary to vitiligo: the possibility of antigen crossover and oxidative stress between the two diseases. Arch Dermatol Res 2016;308:277-281.

7 Irwig MS: Decreased alcohol consumption among former male users of finasteride with persistent sexual side effects: a preliminary report. Alcohol Clin Exp Res 2013;37:18231826.

8 Al-Harbi M: Prevalence of depression in vitiligo patients. Skinmed 2013;11:327-330.
9 Hodes GE, Kana V, Menard C, Merad M, Russo SJ: Neuroimmune mechanisms of depression. Nat Neurosci 2015;18:1386-1393.

10 Caruso D, Abbiati F, Giatti S, Romano S, Fusco L, Cavaletti G, Melcangi RC: Patients treated for male pattern hair with finasteride show, after discontinuation of the drug, altered levels of neuroactive steroids in cerebrospinal fluid and plasma. J Steroid Biochem Mol Biol 2015;146:74-79.

11 Cutolo M, Sulli A, Straub RH: Estrogen metabolism and autoimmunity. Autoimmun Rev 2012;11:460-464.

12 Motofei IG, Rowland DL, Georgescu SR, Mircea T, Baleanu BC, Paunica S: Are hand preference and sexual orientation possible predicting factors for finasteride adverse effects in male androgenic alopecia? Exp Dermatol 2016;25:557-558. 